\title{
Verify Hub Genes of expression profile in aortic dissection
}

Weitie Wang ${ }^{\mathrm{a}}$, Qing Liu ${ }^{\mathrm{b}}$, Yong Wang ${ }^{\mathrm{a}}$, Hulin Piao ${ }^{\mathrm{a}}$, Bo Li ${ }^{\mathrm{a}}$, Zhicheng Zhu ${ }^{\mathrm{a}}$, Dan $\mathrm{Li}^{\mathrm{a}}$, Tiance Wanga ${ }^{\mathrm{a}}$, Rihao $\mathrm{Xu}^{\mathrm{a}}$, Kexiang Liu ${ }^{\mathrm{a} *}$

${ }^{a}$ Department of Cardiovascular Surgery of the Second Hospital of Jilin University, Ziqiang Street 218, Changchun, Jilin, 130041, China

${ }^{\mathrm{b}}$ Graduate School of Medicine and Faculty of Medicine of the University of Tokyo, 7-3-1 Hongo Bunkyo-ku Tokyo, Tokyo, 113-8655, Japan

*Corresponding Author:

Kexiang Liu, M.D. and Ph.D., Department of Cardiovascular Surgery of the Second Hospital of Jilin University, Ziqiang Street 218, Changchun, Jilin, 130041, China. Tel.: 011-86 431-81136791, Fax: 011-86 431-81136791, E-mail address: kxliu64@hotmail.com.

\section{Abstract}

Background: To assess the mRNAs expression profile and explore the hub mRNAs and potential molecular mechanisms in the pathogenesis of human thoracic aortic dissection (TAD). Methodology: mRNA microarray expression signatures of TAD tissues $(n=6)$ and no TAD tissues $(\mathrm{NT} ; \mathrm{n}=6)$ were analyzed by Arraystar human mRNAs microarray. Real-time PCR (qRT-PCR) were used to validate the result of mRNAs microarray. Bioinformatic tools including gene ontology, and Kyoto Encyclopedia of Genes and Genomes pathway analysis were utilized. The protein-protein interaction networks were constructed based on data from the STRING database. Molecular Complex Detection (MCODE) and cytohubba analysis were used to infer the most hug gene and pathways. Results: The top 10 hub genes CDK1, CDC20, CCNB2, CCNB1, MAD2L1, AURKA, C3AR1, NCAPG,CXCL12 and ASPM were identified from the PPI network. Module analysis revealed that TAD 
was associated with cell cycle, oocyte meiosis, p53 signaling pathway, progesterone-mediated oocyte maturation. The qRT-PCR result showed that the expression of all hug genes was significantly increased in TAD samples $(\mathrm{p}<0.05)$. Conclusions: These candidate genes could be used as potential diagnostic biomarkers and therapeutic targets of TAD.

\section{Author summary}

Many basic characteristics underlying the establishment of aortic dissection have not been studied in detail. The presented work sought to understand the pathogenesis of human thoracic aortic dissection by employing bioinformatic tools to explore the hub mRNAs and potential molecular mechanisms of thoracic aortic dissection. Many pathway were thought to have relevant with this disease, but the most important pathway was not define. We used bio-mathematical analysis to explore the potential functions in thoracic aortic dissection and identified the hub genes and explored the intrinsic molecular mechanisms involved in thoracic aortic dissection between two microarray analysis. Finally, we indentified the cell cycle maybe the key pathway in thoracic aortic dissection.

\section{Introduction}

Thoracic aortic dissection (TAD) is a common and a life-threatening aortic disease 1. Despite improvements in medical therapy and surgical or endovascular techniques in recent years, TAD still remains a high morbidity and mortality rate ${ }^{2}$. Owing to the poor results of the existing treatment methods, necessary understanding of the molecular mechanism maybe provided new insights into therapeutic targets for TAD. Many reports show the degradation of extracellular matrix (ECM) and depletion of vascular smooth muscle cell (VSMC) of the aortic wall are the main histopathological findings ${ }^{3-5}$. However, it is remains unclear of the key molecular mechanism of TAD pathogenesis.

In recent years, mRNAs have been reported to participate in the regulation of 
pathophysiological conditions and have been proved to involve in the progression of cardiovascular disease ${ }^{6}$. Nowadays, researches have focused on the Genomewide Association Studies (GWAS) ${ }^{7}$, which could find relevant genetic variants that may be used as potential biomarkers for diagnosis and targeted therapy. Although high-throughput sequencing technology have provided many diverse expressed genes, different expression profile in TAD provided various results and no reliable results have been identified up to now ${ }^{8-10}$. More samples and advanced bio-informatics methods should be used in further study.

In the present study, we used mRNA microarray to acquire differential expression profiles in human TAD tissues and CAD tissues. Subsequently, bio-mathematical analysis were used to explore the potential functions in TAD and identified the hub genes and explore the intrinsic molecular mechanisms involved in TAD. Thus, these data would provide a foundation for new biomarkers and therapeutic targets for human TAD.

\section{RESULTS}

Differential expression profiles of mRNAs in TAD and NT group

Volcano plots revealed that mRNAs were differentially expressed in human TAD aortic tissues through microarray technology (Figure 1). A total of 2834 mRNAs were differentially expressed in TAD group compared with NT group. In total, 1928 mRNAs were up-regulated, and 906 mRNAs were down-regulated (fold change $>2.0$, $\mathrm{P}$ value $<0.05)$. The top ten up- and down-regulated mRNAs are listed in Table 3.

GO Functional Enrichment Analysis.

To know the functions of all DEGs, we used DAVID online tool, the DEGs functions of GO function enrichment were divided into three groups including BP, $\mathrm{CC}$, and MF (Figure 2). As shown in the Figure 2 and Table 4, in the biological processes group, the down-DEGs are mainly enriched in cardiac muscle tissue development, muscle tissue development, muscle structure development, developmental process, and single-multicellular organism process and the up-DEGs 
are mainly enriched inimmune response, response to stress, defense response, immune system process and innate immune response. In the cellular component group, the down- DEGs are mainly enriched in contractile fiber, myofibril, sarcomere, contractile fiber part and cell-substrate junction and the up-DEGs are mainly enriched in cytoplasm, cytoplasmic part, intracellular organelle part, organelle part and membrane-bounded organelle. And in the molecular function group, the down- DEGs are mainly enriched in protein phosphatase regulator activity, phosphatase regulator activity, cytoskeletal protein binding, structural constituent of muscle and actin binding and the up-DEGs are mainly enriched in catalytic activity, protein binding, single-stranded DNA-dependent ATPase activity, ATP binding and adenyl ribonucleotide binding.

Signaling Pathway Analysis.

After the pathway enrichment analysis, down regulated genes were mainly enriched in dilated cardiomyopathy, arrhythmogenic right ventricular cardiomyopathy, hypertrophic cardiomyopathy, adrenergic signaling in cardiomyocytes and vascular smooth muscle contraction. And up regulated genes were mainly enriched in DNA replication, phagosome, cell cycle, staphylococcus aureus infection and lysosome (Figure 3,4).

PPI Network and Modular Analysis.

All the DEGs (fold change $>4.0$ ) were analysis using STRING database. Then we put these data into Cytoscape software to construct a PPI network which containing 433 nodes and 348 edges (Figure 5). In these DEGs, 10 hub genes including CDK1, CDC20, CCNB2, CCNB1, MAD2L1, AURKA, C3AR1, NCAPG,CXCL12, ASPM after calculating. In these 10 hub genes, CDK1 present with the highest degree (degree $=44)$. The Cytoscape plugin MCODE shows the top three module with 15.429,14.857 and 10.000 score respectively (Figure 6). Then the genes in these three module were performed with functional enrichment analyses. Pathway enrichment analysis showed that Module 1 is mainly relevant with cell cycle oocyte meiosis, p53 
signaling pathway, progesterone-mediated oocyte maturation. Module 2 is mainly associated with chemokine signaling pathway, cytokine-cytokine receptor interaction, rheumatoid arthritis, NF-kappa B signaling pathway, neuroactive ligand-receptor interaction. Module 3 is mainly associated with leukocyte transendothelial migration. Validation by qRT-PCR of differentially expressed mRNAs

To validate microarray results, the expression levels of top 10 hub genes were determined in ascending aortic samples of thoracic aortic dissection and no thoracic aortic dissection using qRT-PCR. The verification result showed that the expression levels of the 10 hub genes were significantly increased in thoracic aortic dissection samples $(\mathrm{p}<0.05)$ (Figure 7). All validations are consistent with the microarray data and analytical results in this study.

\section{Disscussion}

TAD is a life-threatening event that carries a high mortality rate ${ }^{1}$. Patients always complain with acute chest and back pain and are often misdiagnosed as acute myocardial infarction ${ }^{15}$. Surgical treatment seems to the best method of this disaster disease ${ }^{16}$. However, the traditional surgical treatment of TAD is very complex and time-consuming with high mortality and poor prognosis. Therefore it is necessary to research of the new biomarkers and therapeutic targets. Up to now, the molecular mechanism of this severe disease remains unclear. TAD such as Marfan syndrome and Ehlers-Danlos syndrome had been confirmed with deficiency of the glycoprotein ${ }^{17}$ and abnormal type-III precollagen ${ }^{18}$ respectively. However, most patients with TAD do not exhibit such explicit syndromes. These TAD patients always complain with hypertension, atherosclerosis and trauma ${ }^{15}$. In addition, lots of studies had pointed that degradation of ECM and depletion of VSMC play an important role in un-heredity TAD ${ }^{19}$. So studies are primarily focused on the protein-coding genes of $\mathrm{ECM}^{20}$ and VSMC such as COL3A1, FBN1, LOX, FLNA, ACTA2, MYH11. These protein-coding genes play role in pathological processes of TAD. However, the key pathogenesis of the TAD has not been confirmed up to now. 
Modern molecular biology believe that the disease was induced by the changing of various gene expression profiles of tissues or cells. Collection, summary and analysis of the large numbers of these gene can help us to understand the mechanism of the diseases. High-throughput sequencing technology make the identity of the changing gene in tissue available and have been widely used to predict potential targets gene. Up to now, there are many studies have been performed and thousands of differentially expressed genes had been screened. However, different gene profile are various with each study ${ }^{8-10}$. So more further analysis should be carried out and hub genes and pathway should be identify.

This study identify 2834 mRNAs DEGs which included 1928 upregulated DEGs and 906 downregulated DEGs (fold change $>2.0$, P value $<0.05$ ). Like other studies ${ }^{8-10}$, we classified into three groups including BP,CC, PF by GO terms. GO functional enrichment analysis showed that immune response, response to stress, defense response and immune system process, which was consistent with other studies. Inflammatory and immunological may both probably lead to the degree of vascular damage of aortic wall ${ }^{21-23}$. Reports had presented that inflammatory mechanisms participated in medial degeneration of aortic dissection tissue. The macrophages and activated $\mathrm{T}$ lymphocytes were also found in the dissection tissue. T helper 2 response was reported to have relevant with the growth of aneurysms ${ }^{24-25}$. hyper-expression IL-6 and IL-8 in aortic dissection was also present that immunologic pathways were critical in the aortic wall damage ${ }^{26}$. In addition, neutrophils, CD8+, CD28-,IL-6, TNF- $\alpha$, IL-8, and MCP-1 suggestted that cytotoxic and innate cells were mainly relevant with the pathogenesis of $\mathrm{TAD}^{27}$. T helper lymphocytes and activated macrophages, mast cells, T and B lymphocytes that immune system process and inflammatory pathways were involved in the weakening of the aortic wall.

Furthermore, the enriched KEGG pathways of downregulated genes were mainly enriched in dilated cardiomyopathy, arrhythmogenic right ventricular cardiomyopathy, hypertrophic cardiomyopathy, adrenergic signaling in cardiomyocytes and vascular smooth muscle contraction. VSMC was the major cell in aortic media. The function of VSMC such as proliferation and migration acted critical biomechanical properties 
of the aortic wall. It was affluence by many factor including lymphocytes induce apoptosis of VSMCs and synthesis of MMPs. So many studies aim to research the function of VSMC. Talin-1 had been reported to main exist in aortic media and it was significant downregulation in AD aortic tissue. Further study confirmed that Talin-1 regulating VSMC proliferation and migration and finally caused of pathologic vascular remodeling to change vascular media structure and function and lead to AD 28. Many gene such as YAP1, Sirtuin-1, PCSK9, polycystin-1, brahma-related gene 1 had also been reported to associated with the pathophysiologic processes of aortic dissection through influence proliferation and migration of VSMC ${ }^{29-31}$.

Like previous high-throughput sequencing studies, we also got many relevant pathways through analysising, but we can not sure which one is the most relevant mechanism about TAD and the most hug gene about $\mathrm{AD}$ is also not sure. So we carry out some further analysis such as PPI Network and Modular Analysis including STRING database, cytohubba analysis and MCODE analysis which is mainly widely used in infer the most hug gene and pathways ${ }^{32}$. In our study, we choose gene which is different express up to 4 fold in order to reduce the scope. The cytohubba analysis show that the top ten hug genes are CDK1, CDC20, CCNB2, CCNB1, MAD2L1, AURKA, C3AR1, NCAPG,CXCL12, ASPM. These gene shows the cell cycle seem to play an important role in TAD. So the Cytoscape plugin MCODE was used to analysis from another aspect. After calculating, the top module including 22 nodes and 162 edges. We choose the 22 nodes for functional enrichment analyses. And the cell cycle was the most relevant pathway and also include 7 hug genes which previous forcast. So we aim to analysis the hug gene CDK1 and cell cycle in TAD.

CDK1 is the founding member of the CDK family in human cells ${ }^{33}$. It can conserve across all leukocytes and is the only essential cell cycle CDK in human cells. Study reported that this important member of CDK family is required for successful completion of M-phase. In addition, the conserved nature and remodelled function make this molecular more special. In cell cycle, CDK1 is always act it function combine with cyclin A and cyclin B. So CDK1 can regulate the cell cycle from GI to S phase because it partner-cyclin A- is first expressed during late G1 where it initially 
binds to CDK2 and promotes S-phase ${ }^{34}$. As the cyclin-dependent kinase (CDK) family in association with partner cyclin proteins will mediated the progression of cell cycle, CDK1 inhibition will lead the initiates adhesion remodeling in preparation for entry into mitosis and reveal an intimate link between the cell cycle machinery and cell - ECM adhesion. So the toppest degree hug gene is CDK1 which is mean the cell cycle will act as the key role in TAD. The Cytoscape plugin MCODE result also show the first progosis passway is cell cycle. The two analysis method are consistent and also in accordence with previous studies ${ }^{8-10}$.

The endothelial cells and SMC are important in maintaining of vascular tone in blood vessel. Previous study confirm that restricting cell cycle of endothelial cell and SMC may lead to disorder of vascular remodeling as an antecedent to the pathological sequelae of cardiovascular disease ${ }^{35}$. The SMC function changing are two main pathogenesis of TAD. SMC progression and proliferation are critical for the development of TAD. So most studies aim to research the cell cycle of SMC ${ }^{36}$. Arrests proliferating SMCs in G0/G1 and G2/M-phases and inhibits cyclin B1 expression and cdk1 activity (essential for G2-to-M progression) may protect vascular-proliferative diseases. Other investigations also revealed an important role of VSMCs proliferative in aortic tissue degeneration, which has been believed as the initiation of pathologic remodeling in $\mathrm{TAD}^{37}$. it is obvious that the abnormal proliferation and migration of VSMCs and changing of cell cycle have been proved to the considered as the potentially main cause of pathological vascular remodeling through undermining the vasculature stability and finally lead to vascular disease. many studies aim to research the VSMCs from dissected aorta and indentidy this SMC can proliferated more rapidly than normal VSMCs tissues, and the genes participate in proliferation exhibited an increased expression. In our study, the 10 hug genes are all up expression which show the up regulaition defferent expression will act an important role in TAD which is coninsistance with previous study.

In summary, by means of high-throughput sequencing and data processing as well as qRT-PCR validation, the hub genes including CDK1, CDC20, CCNB2, 
CCNB1, AURKA, NCAPG and ASPM may have the potential to be used as drug targets and diagnostic markers of TAD. Cell cycle maybe the key pathway in TAD. However, there were still some limitations: normal aortic tissue used as control group maybe more accuracy. Further experimental studies with larger sample size need to confirm cell cycle pathway in TAD.

\section{Methods}

Tissue collection

This study was conducted in accordance with the Declaration of Helsinki and was approved by the Ethics Committee of the Second Hospital of Jilin University. Ascending aortic specimens near the intimal tear were obtained from TAD patients undergoing surgical repair $(n=6)$ if the informed consent was obtained. Aortas specimens derived from patients without aortic diseases undergoing coronary artery bypass ( $n=6$ ) graft were obtained as no TAD (NT) group if the informed consent was obtained. General information of all patients was shown in Table 1. There were no significant differences in age, gender, obesity, smoking between the two groups. Patients diagnosed with TAD were confirmed by the computed tomography angiography (CTA) and excluded from any heredity TAD. All the specimens were immediately frozen in liquid nitrogen, and preserved at $-80^{\circ} \mathrm{C}$ for microarray analysis and qRT-PCR or further usage.

RNA extraction and quality control

Tissue RNA from TAD and NT group was extracted according to the manufacturer's instructions by TRIzol reagent (Invitrogen, NY, USA). NanoDrop ND- 1000 (Thermo Fisher Scientific, Wilmington, DE, USA) and Agilent 2100 Bioanalyzer (Agilent Technologies) were used to check the integrity and concentration of the RNA samples. All the qualified samples were stored at $-80^{\circ} \mathrm{C}$ for future experiments. 
Microarray analysis

There are 12 samples (6 TAD and 6 NT) were used for microarray analysis by Arraystar Human mRNA microarray which contained 14596 mRNAs probes. Sample preparation and array hybridization were performed according to the Agilent One-Color Microarray-Based Gene Expression Analysis protocol (Agilent). Briefly, mRNA was purified from total-RNA (RNeasy Mini Kit, Qiagen) after removal of rRNA and transcribed into fluorescent cRNA without 3' bias along the entire length of the transcripts with a random priming method. Quick-Amp Labeling Kit (Agilent, USA) was used for sample labeling. Then NanoDrop ND-1000 was used to check the specific activity and concentration of the labeled cRNAs. Then hybridization was performed in an Agilent Hybridization Oven. Data normalization and processing was performed using the GeneSpring GX v12.1 software package (Agilent). After quantile normalization of the raw data, mRNAs samples had flags in Present or Marginal ("All Targets Value") were chosen for further data analysis. The differentially expressed mRNAs were statistical significance with the change in threshold values was $>2.0$ or $<-2.0$ fold between the two groups and Benjamini-Hochberg corrected $\mathrm{P}<0.05$.

Gene Ontology (GO) and Pathway Enrichment Analyses.

DAVID (the Database for Annotation, Visualization, and Integrated Discovery) online bio-informatics database is an analysis tools of biological data to integrates and provide information for biological function and protein list ${ }^{11}$. This tool was used in this study to provide GO enrichment and Kyoto Encyclopedia of Genes and Genomes (KEGG) pathway analysis. GO analysis included categories of cellular component (CC), biological processes (BP) and molecular function (MF). Pathway analysis is a functional analysis that maps genes to KEGG pathways. And gene count $>2$ and $p<$ 0.05 were set as the cutoff point.

Integration of Protein-Protein Interaction (PPI) Network Analysis.

STRING (https://string-db.org/cgi/input.pl) is an online database resource search tool which can provide analysis of interacting genes including physical and functional 
associations ${ }^{12}$. In this study, the STRING online tool was used to construct a PPI network of upregulation and downregulation diferentially expressed genes (DEGs), with a confidence score $>0.7$ defined as significant. Then the interaction data were typed into the Cytoscape software ${ }^{13}$ to structure a PPI network. Based on the above data, we used Molecular Complex Detection (MCODE) ${ }^{14}$, a built-in APP in Cytoscape software, to analyze the interaction relationship of the DEGs encoding proteins and screening hub gene. The parameters of network scoring and cluster finding were set as follows: degree cutoff $=2$, node score cutoff $=0.2, \mathrm{k}$-core $=2$, and $\max$ depth $=100$.

Quantitative reverse transcription-PCR (qRT-PCR)

Validation and Statistical Analysis. qRT-PCR was used to verify the core genes. Total RNA was reverse-transcribed to cDNA using PrimeScript RT reagent Kit with gDNA Eraser (TaKaRa, Japan) according to the manufacturer's instructions. Primer 5.0 software (PREMIER Biosoft, Palo Alto, CA, USA) was used to design primers, and a QuantStudio 7 Flex real-time PCR system (Applied Biosystems, Carlsbad, CA, USA) was used. All primers used in this study were listed in Table 2. All samples were normalized to GAPDH. And the relative expression levels of each gene were calculated using $2-\Delta \mathrm{Ct}$ methods.

\section{Statistical analysis}

The data were presented as the mean \pm standard deviation. Statistical analyses were performed using SPSS version 20.0 (SPSS Inc., Chicago, IL, USA). The raw data were preprocessed by affy package in R software and limma package in R software . Comparisons between groups were performed using unpaired Student's t-test. Fisher's exact test was used to evaluate the significance of GO terms and Pathway identifiers enrichment. The false discovery rate (FDR) controlling was used to correct p-value. FDR and P-value less than 0.05 was considered statistically significant. 


\title{
Acknowledgments
}

\author{
None
}

\section{References}

[1] Lio A, et al. Influence of Body Mass Index on Outcomes of Patients Undergoing Surgery for Acute Aortic Dissection: A Propensity-Matched Analysis.Tex Heart Inst J. 46, 7-13 (2019).

[2] Nienaber CA, Clough RE. Management of acute aortic dissection. Lancet. 385, 800-811 (2015).

[3] Zhao Z, et al. HSP90 inhibitor 17-DMAG effectively alleviated the progress of thoracic aortic dissection by suppressing smooth muscle cell phenotypic switch. Am J Transl Res. 11, 509-518 (2019).

[4] Cheng J, Zhou X, Jiang X, Sun T. Deletion of ACTA2 in mice promotes angiotensin II induced pathogenesis of thoracic aorticaneurysms and dissections. J Thorac Dis. 8, 4733-4740 (2018).

[5] An Z, et al. Interleukin-6 downregulated vascular smooth muscle cell contractile proteins via ATG4B-mediated autophagy in thoracic aortic dissection. Heart Vessels. 12, 1523-1535 (2017).

[6] Herman AB, Autieri MV. Inflammation-regulated mRNA stability and the progression of vascular inflammatory diseases. Clin Sci (Lond). 131, 2687-2699 (2017).

[7] McCoy TH Jr, Fragomen AT, Hart KL, Pellegrini AM, Raskin KA, Perlis RH. Genomewide Association Study of Fracture Nonunion Using Electronic Health Records. JBMR Plus. 20, $23-28$ (2018).

[8] Kimura N, et al. Gene expression profiling of acute type A aortic dissection combined with in vitro assessment. Eur J Cardiothorac Surg. 52, 810-817 (2017).

[9] Li Y, et al. LncRNA and mRNA interaction study based on transcriptome profiles reveals potential core genesin the pathogenesis of human thoracic aortic dissection. Mol Med Rep. 18, 3167-3176 (2018). .

[10] Ohno-Urabe S, et al. Role of Macrophage Socs3 in the Pathogenesis of Aortic Dissection. J Am Heart Assoc. 17, e007389 (2018).

[11] D. W. Huang, et al. Te DAVID gene functional classifcation tool: a novel biological module-centric algorithm to functionally analyze large gene lists. Genome Biology. 9, R183 (2017).

[12] D. Szklarczyk, et al.Te STRING database in 2011: functional interaction networks of proteins, globally integrated and scored. Nucleic Acids Research. 39, 561-568 (2011).

[13] P. Shannon, A. Cytoscape: a sofware Environment for integrated models of biomolecular interaction networks. Genome Research. 13, 2498 - 2504 (2003).

[14] G. D. Bader, et al. An automated method for fnding molecular complexes in large protein interaction networks. BMC Bioinformatics. 4, 1-2 (2003).

[15] Gawinecka J, Schönrath F, von Eckardstein A. Acute aortic dissection: pathogenesis, risk factors and diagnosis. Swiss Med Wkly. 147, 14489 (2017).

[16] Berger T, et al. Multicentre experience with two frozen elephant trunk prostheses in the treatment of acute aortic dissection. Eur J Cardiothorac Surg. 7, 037 (2019).

[17] Wang F, Li B, Lan L, Li L. C596G mutation in FBN1 causes Marfan syndrome with exotropia in a Chinese family. Circ. Res. 21, 194-200 (2015).

[18] Reinstein E, et al. Ehlers-Danlos syndrome type VIII is clinically heterogeneous disorder associated primarily with periodontal disease, and variable connective tissue features. Eur J Hum Genet. 21, 233-236 (2013).

[19] Mallat Z, Tedgui A, Henrion D. Role of Microvascular Tone and Extracellular Matrix Contraction in the Regulation of Interstitial Fluid: Implications for Aortic Dissection. Arterioscler Thromb Vasc Biol. 36, 
1742-1746 (2016).

[20] Li Z, et al. Variants of genes encoding collagens and matrix metalloproteinase system increased the risk of aortic dissection.Sci China Life Sci. 60, 57-65 (2017).

[21] He R. Characterization of the infl ammatory and apoptotic cells in the aortas of patients with ascending thoracic aortic aneurysms and dissections. J Thorac Cardiovasc Surg. 131, 671-678 (2006).

[22] Luo F, Zhou XL, LI JJ, Hui RT. Inflammatory response is associated with aortic dissection. Ageing Res Rev. 8, 31-35 (2009)

[23] Weis-Muller BT, et al. Gene expression in acute Stanford type A dissection: a comparative microarray study. J Transl Med. 4, 29-44 (2006).

[24] Schonbeck U, Sukhova GK, Gerdes N, Libby P. Th2 predominant immune responses prevail in human abdominal aortic aneurysm. Am J Pathol, 161, 499 - 506 (2002).

[25] Schulte S, Sukhova GK, Libby P. Genetically programmed biases in Th1 and Th2 immune responses modulates atherogenesis. Am J Pathol. 172, 1500-1508. (2008).

[26] Lindeman JH, et al. Enhanced expression and activation of pro-infl ammatory transcription factors distinguish aneurysmal from atherosclerotic aorta: IL-6 and IL-8-dominated infl ammatory responses prevail in the human aneurysm. Clin Sci (Lond). 114, 687-697 (2008).

[27] del Porto F, et al. Inflammation and immune response in acute aortic dissection. Ann Med. 42, 622-699 (2010).

$$
\text { Wei } \mathrm{X} \text {, }
$$$$
\text { et }
$$

Downregulation of Talin-1 expression associates with increased proliferation and migration of vascular sm ooth muscle cells in aortic dissection. BMC Cardiovasc Disord. 20, 162 (2017).

[29] Iida Y, et al. Ectopic Expression of PCSK9 by Smooth Muscle Cells Contributes to Aortic Dissection. Ann Vasc Surg. 48, 195-208 (2018).

$$
\text { Fry }
$$

$\mathrm{JL}$,

et

al.

Vascular Smooth Muscle Sirtuin-1 Protects Against Aortic Dissection During Angiotensin II-Induced Hype rtension. J Am Heart Assoc, 16, e002384 (2015).

$$
\text { Liao }
$$

WL

et

al.

Brahma-related gene 1 inhibits proliferation and migration of human aortic smooth muscle cellsby directly up-regulating Ras-related associated with diabetes in the pathophysiologic processesof aortic dissection. $J$ Thorac Cardiovasc Surg. 150, 1292-301 (2015).

Integration of Gene Expression Profile Data to Screen and Verify Hub Genes Involved in Osteoarthritis. Biomed Res Int. 14, 9482726 (2018).

[33] Brown NR, et al. CDK1 structures reveal conserved and unique features of the essential cell cycle CDK.Nat Commun.13, 6769 (2015).

[34] Diril, M. K., et al. Cyclin-dependent kinase 1 (Cdk1) is essential for cell division and suppression of DNA re-replication but not for liver regeneration. Proc. Natl Acad. Sci. 109, $3826-3831$ (2012). doi: 10.3389/fmolb.2017.00038.

$$
\text { Hsieh }
$$$$
\mathrm{TC}, \mathrm{Lu}
$$$$
\text { X, Guo }
$$

Differential regulation of proliferation, cell cycle control and gene expression in cultured humanaortic and pulmonary artery endothelial cells by resveratrol. Int J Mol Med. 26, 743-749 (2010).

[36] Barchiesi F, Jackson EK, Fingerle J, Gillespie DG, Odermatt B, Dubey RK. 2-Methoxyestradiol, an estradiol metabolite, inhibits neointima formation and smooth muscle cellgrowth via double blockade of the cell cycle.Circ Res. 99, 266-274 (2006). 
[37] Zhu L, et al. Mutations in myosin heavy chain 11 cause a syndrome associating thoracic aortic aneurysm/aortic dissection and patent ductus arteriosus. Nat Genet. 38, 343 - 349 (2006).

\section{Figure legend}

Figure 1. Comparison of mRNA expression profile between the TAD samples and NT samples.

Comparison of mRNA expression profile between the TAD samples and NT

samples. (A) The box plot is a convenient method to quickly compare the distribution of mRNAs. After normalization, the distributions of $\log 2$ ratios among the tested samples are almost similar. (B) The scatterplot is a visualization method that is useful for assessing the variation between the TAD and control tissues compared by microarrays. The values of $\mathrm{X}$ and $\mathrm{Y}$ axes in the scatterplot are averaged normalized values in each group ( $\log 2$ scaled). The green plot are decrease and red plot are upgrade different gene.(C)diferentially expressed genes can be effectively divided into TAD and NT groups. Red indicates that the gene that is upregulated and green represents down-regulated genes.

Figure 2. Gene Ontology analysis classified the differentially expressed genes into 3 groups

molecular function, biological process, and cellular component.

Figure 3. Kyoto Encyclopedia of Genes and Genomes enrichment analysis of the pathways.

The gradual color represents the $\mathrm{P}$ value; the size of the black spots represents the gene number.

Figure 4. Signifcantly up-regulated and down-regulated pathways.

Figure 5. PPI network constructed with the differentially expressed genes

PPI network constructed with the differentially expressed genes. Red nodes represent hub GENE analysis by cytohubba. 
Figure 6 : The three most significance modules.

Red nodes represent hug GENE analysis by cytohubba.

Figure 7. Validation of the mRNA microarray results by real-time qPCR.

mRNA microarray result was verified by real-time qPCR between the TAD group $(n=10)$ and the NT group $(n=10)$. All samples were normalized to the expression of GAPDH, and the relative expression levels of each gene were analyzed using the $2-\triangle \triangle \mathrm{Ct}$ method. $* \mathrm{P}<$ $0.05, * * \mathrm{P}<0.01$

\section{Contributions}

W.T.W, B.L, H.L.P ,Q.L and Y.W wrote the paper. Z.C.Z, D.L, T.C.W, R.H.X, and K.X.L checked the References. All authors reviewed the final manuscript.

\section{Data availability}

Data available on request for the Ethics Committee of the Second Hospital of Jilin University .

\section{Dual publication}

The elements in the manuscript have not been published or are under consideration for publication elsewhere.

\section{Financial disclosure}

This work was supported by the project supported by the Bethune Medical Department Doctoral Postgraduate Excellent Talents Training Program Project of Jilin Province, China (Grant no. 20181201), the Project of Direct Health Project of Jilin Provincial, China (Grant no. 20170101), and Project of Direct Health Project of Jilin Provincial, China (Grant no. 20180101). The funders had no role in study design, data collection and analysis, decision to publish, or preparation of the manuscript.

\section{Additional Information}


Competing financial interests: The authors declare no competing financial and/or non-financial interests in relation to the work described.

\begin{abstract}
Abbreviation
thoracic aortic dissection (TAD); extracellular matrix (ECM); vascular smooth muscle cell (VSMC); Genomewide Association Studies (GWAS); no thoracic aortic dissection (NT); computed tomography angiography (CTA); Gene Ontology (GO); Kyoto Encyclopedia of Genes and Genomes (KEGG); cellular component (CC); biological processes (BP); molecular function (MF); Protein-Protein Interaction (PPI); diferentially expressed genes (DEGs); Molecular Complex Detection (MCODE); Quantitative reverse transcription-PCR (qRT-PCR); false discovery rate (FDR); cyclin-dependent kinase (CDK)
\end{abstract}

\title{
Table legend
}

Table 1 General information of all patients

Table 2: The primers of top 10 hub genes

Table 3: The top ten up- and down-regulated mRNAs

Table 4 The significant enriched analysis of differentially expressed genes in thoracic aortic dissection

Table 1 General information of all patients

TAD group NT group $\quad$ P value




\begin{tabular}{|llll|} 
& $(\mathrm{n}=6)$ & $(\mathrm{n}=6)$ & \\
\hline Age $($ years old $)$ & $55.61 \pm 9.23$ & $57.53 \pm 10.61$ & 0.7450 \\
Male & $3(50.00 \%)$ & $3(50.00 \%)$ & - \\
Obesity $(\mathrm{BMI}>25 \mathrm{~kg} / \mathrm{m} 2)$ & $2(33.33 \%)$ & $3(50.00 \%)$ & 0.5582 \\
Smoking & $2(33.33 \%)$ & $4(66.67 \%)$ & 0.2482 \\
NYHA class III-IV & $1(16.67 \%)$ & $2(33.33 \%)$ & 0.5055 \\
Hypertension & $4(66.67 \%)$ & $3(50.00 \%)$ & 0.5582 \\
Diabetes mellitus & $1(16.67 \%)$ & $3(50.00 \%)$ & 0.2207 \\
Chronic renal dysfunction & 0 & 0 & - \\
\hline
\end{tabular}

TAD: Thoracic aortic dissection; NT: no TAD; NYHA : New York Heart Association

Table 2: The primers of top 10 hub genes.

\begin{tabular}{|l|l|l|}
\hline Gene name & Forward primer & Reverse primer \\
\hline CDK1 & TTGGCTGCTTTGAAAGTCTACG & GGTATGGTAGATCCGCGCTAAA \\
\hline CDC20 & ATGCGCCAGAGGGTTATCAG & AGGATGTCACCAGAGCTTGC \\
\hline CCNB2 & TCCAAAGGGTCCTTCTCCCA & TTGCAGAGCAAGGCATCAGA \\
\hline CCNB1 & GAAACGCATTCTCTGCGACC & AGCATTAATTTTCGAGTTCCTGGT \\
\hline MAD2L1 & CGTGCTGCGTCGTTACTTTT & GCCGAATGAGAAGAACTCGG \\
\hline AURKA & GGATATCTCAGTGGCGGACG & GCAATGGAGTGAGACCCTCT \\
\hline C3AR1 & CAGTGAGGAGCTCACACGTT & TAAGAGCCCCTGCTTGTTGG \\
\hline NCAPG & GCCCATTGTTACTGTTGGTGTT & TGCAATGTTTCAGCATCATTCTTCT \\
\hline CXCL12 & CTGTGCCCTTCAGATTGTAGCC & AAAGTGTGCAAAACAAAGCCCT \\
\hline ASPM & AGAGTTAATGCAGCACTCGTCA & CCTCCACATAGCCTGAATAAGTGA \\
\hline GAPDH & CGGACCAATACGACCAAATCCG & AGCCACATCGCTCAGACACC \\
\hline
\end{tabular}

Table 3: The top ten up- and down-regulated mRNAs .

\begin{tabular}{llllll}
\hline $\begin{array}{l}\text { Up-regulated } \\
\text { GeneSymbol }\end{array}$ & \multicolumn{1}{c}{ FDR } & \multicolumn{1}{c}{ P-value } & Gown-regulated \\
\hline MARCO & 258.8940442 & $9.67102 \mathrm{E}-06$ & ATXN3L & 108.3473569 & $2.30333 \mathrm{E}-05$ \\
CHI3L2 & 135.8107372 & 0.00011992 & SLC51B & 83.5617528 & $2.52205 \mathrm{E}-06$ \\
CCL18 & 72.0452345 & $3.67575 \mathrm{E}-05$ & GYS1 & 60.3188012 & $7.75796 \mathrm{E}-06$ \\
SPP1 & 62.9254504 & 0.000147395 & KITLG & 60.045535 & $6.2348 \mathrm{E}-06$ \\
SAA2 & 53.30226 & $2.78619 \mathrm{E}-05$ & C8orf12 & 52.4563995 & $7.96212 \mathrm{E}-05$ \\
APOC1 & 42.1502422 & $7.24249 \mathrm{E}-05$ & OR6A2 & 52.3701259 & $3.53724 \mathrm{E}-05$ \\
SERPINA3 & 33.7417914 & $2.36918 \mathrm{E}-07$ & AC093323.1 & 47.6062725 & $2.71921 \mathrm{E}-06$ \\
MT1G & 33.6791872 & 0.000460576 & ITLN1 & 47.4071743 & $1.01876 \mathrm{E}-07$ \\
CHRDL2 & 33.6103171 & $1.26255 \mathrm{E}-05$ & ZNF367 & 46.8660148 & $6.64981 \mathrm{E}-06$ \\
RRM2 & 32.9976749 & 0.000779975 & OFCC1 & 41.6471558 & $6.39121 \mathrm{E}-05$ \\
\hline
\end{tabular}


Table 4 The significant enriched analysis of differentially expressed genes in thoracic aortic dissection.

\begin{tabular}{|c|c|c|c|c|c|}
\hline Expression & Category & Term & Description & Gene Count & P-Value \\
\hline \multirow{16}{*}{$\begin{array}{c}\text { DOWN-DE } \\
\text { Gs }\end{array}$} & $\mathrm{BP}$ & $\begin{array}{l}\text { cardiac muscle tissue } \\
\text { development }\end{array}$ & GO:0048738 & 30 & $1.43 \mathrm{E}-10$ \\
\hline & $\mathrm{BP}$ & $\begin{array}{l}\text { muscle } \\
\text { development }\end{array}$ & GO:0060537 & 43 & $2.41 \mathrm{E}-10$ \\
\hline & $\mathrm{BP}$ & $\begin{array}{ll}\text { muscle } & \text { structure } \\
\text { development } & \end{array}$ & GO:0061061 & 58 & $4.80 \mathrm{E}-10$ \\
\hline & $\mathrm{BP}$ & developmental process & GO:0032502 & 320 & $1.23 \mathrm{E}-09$ \\
\hline & $\mathrm{BP}$ & $\begin{array}{l}\text { single-multicellular } \\
\text { organism process }\end{array}$ & GO:0044707 & 323 & 2.13E-09 \\
\hline & $\mathrm{CC}$ & contractile fiber & GO:0043292 & 32 & $6.27 \mathrm{E}-10$ \\
\hline & $\mathrm{CC}$ & myofibril & GO:0030016 & 30 & $2.86 \mathrm{E}-09$ \\
\hline & $\mathrm{CC}$ & sarcomere & GO:0030017 & 28 & $3.41 \mathrm{E}-09$ \\
\hline & $\mathrm{CC}$ & contractile fiber part & GO:0044449 & 29 & $5.76 \mathrm{E}-09$ \\
\hline & $\mathrm{CC}$ & cell-substrate junction & GO:0030055 & 41 & $1.51 \mathrm{E}-07$ \\
\hline & MF & protein phosphatase & & & \\
\hline & & regulator activity & GO:0019888 & 13 & $1.20 \mathrm{E}-05$ \\
\hline & MF & phosphatase regulator & & & \\
\hline & & activity & GO:0019208 & 14 & $1.39 \mathrm{E}-05$ \\
\hline & MF & cytoskeletal protein & & & \\
\hline & & binding & GO:0008092 & 57 & $2.60 \mathrm{E}-04$ \\
\hline \multirow{17}{*}{ UP-DEGs } & $\mathrm{MF}$ & $\begin{array}{l}\text { structural constituent of } \\
\text { muscle }\end{array}$ & GO:0008307 & 8 & $2.74 \mathrm{E}-04$ \\
\hline & MF & actin binding & GO:0003779 & 32 & $2.77 \mathrm{E}-04$ \\
\hline & $\mathrm{BP}$ & immune response & GO:0006955 & 277 & $4.29 \mathrm{E}-26$ \\
\hline & $\mathrm{BP}$ & response to stress & GO:0006950 & 521 & $1.53 \mathrm{E}-24$ \\
\hline & $\mathrm{BP}$ & defense response & GO:0006952 & 263 & $1.99 \mathrm{E}-23$ \\
\hline & $\mathrm{BP}$ & immune system process & GO:0002376 & 371 & $2.30 \mathrm{E}-22$ \\
\hline & $\mathrm{BP}$ & innate immune response & GO:0045087 & 157 & $5.71 \mathrm{E}-17$ \\
\hline & $\mathrm{CC}$ & cytoplasm & GO:0005737 & 1233 & $7.68 \mathrm{E}-33$ \\
\hline & $\mathrm{CC}$ & cytoplasmic part & GO:0044444 & 983 & $4.18 \mathrm{E}-31$ \\
\hline & $\mathrm{CC}$ & intracellular organelle part & GO:0044446 & 984 & $5.62 \mathrm{E}-28$ \\
\hline & $\mathrm{CC}$ & organelle part & GO:0044422 & 995 & $1.89 \mathrm{E}-26$ \\
\hline & $\mathrm{CC}$ & membrane-bounded & & & \\
\hline & & organelle & GO:0043227 & 1323 & $6.40 \mathrm{E}-24$ \\
\hline & MF & catalytic activity & GO:0003824 & 695 & $1.76 \mathrm{E}-12$ \\
\hline & MF & protein binding & GO:0005515 & 1006 & 3.26E-11 \\
\hline & MF & single-stranded & & & \\
\hline & & $\begin{array}{l}\text { DNA-dependent ATPase } \\
\text { activity }\end{array}$ & GO:0043142 & 10 & 4.99E-09 \\
\hline
\end{tabular}


bioRxiv preprint doi: https://doi.org/10.1101/627125; this version posted May 3, 2019. The copyright holder for this preprint (which was not certified by peer review) is the author/funder, who has granted bioRxiv a license to display the preprint in perpetuity. It is made available under aCC-BY 4.0 International license.

MF

ATP binding

GO:0005524

208

2.82E-08

MF

adenyl ribonucleotide

binding

GO:0032559

212

$3.10 \mathrm{E}-08$ 
A

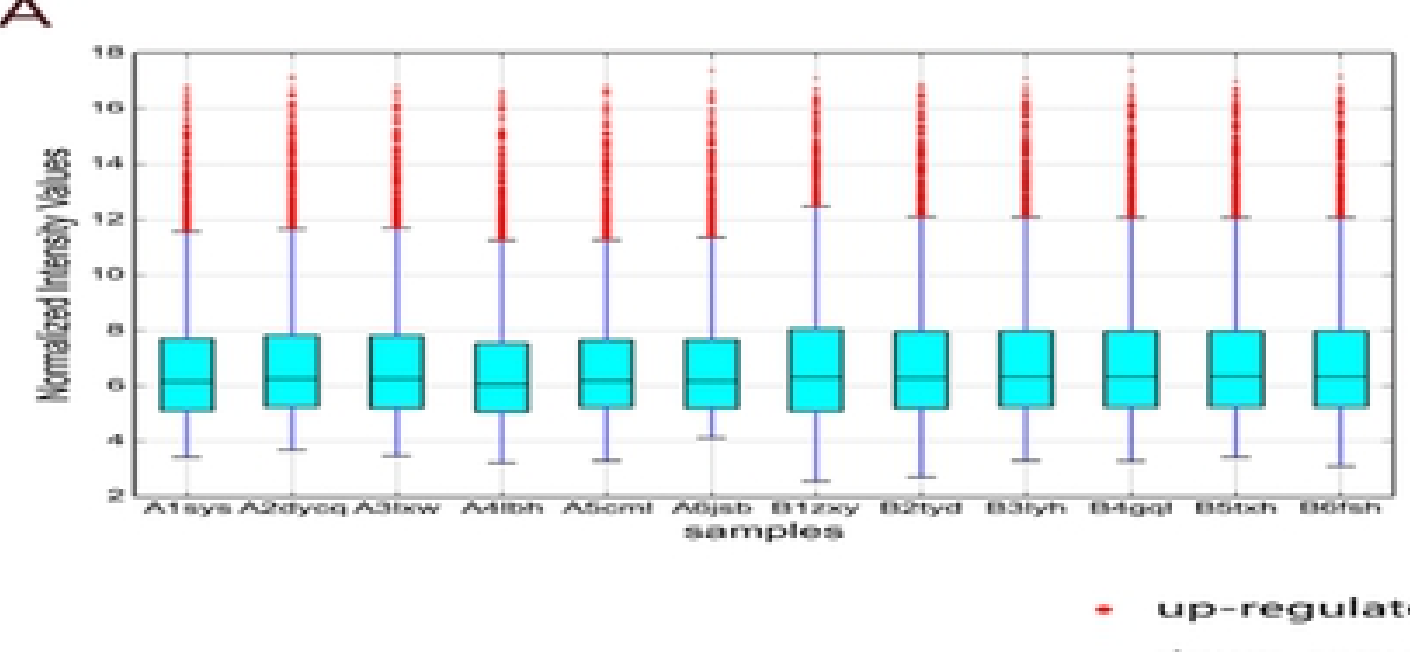

- up-regulated

B

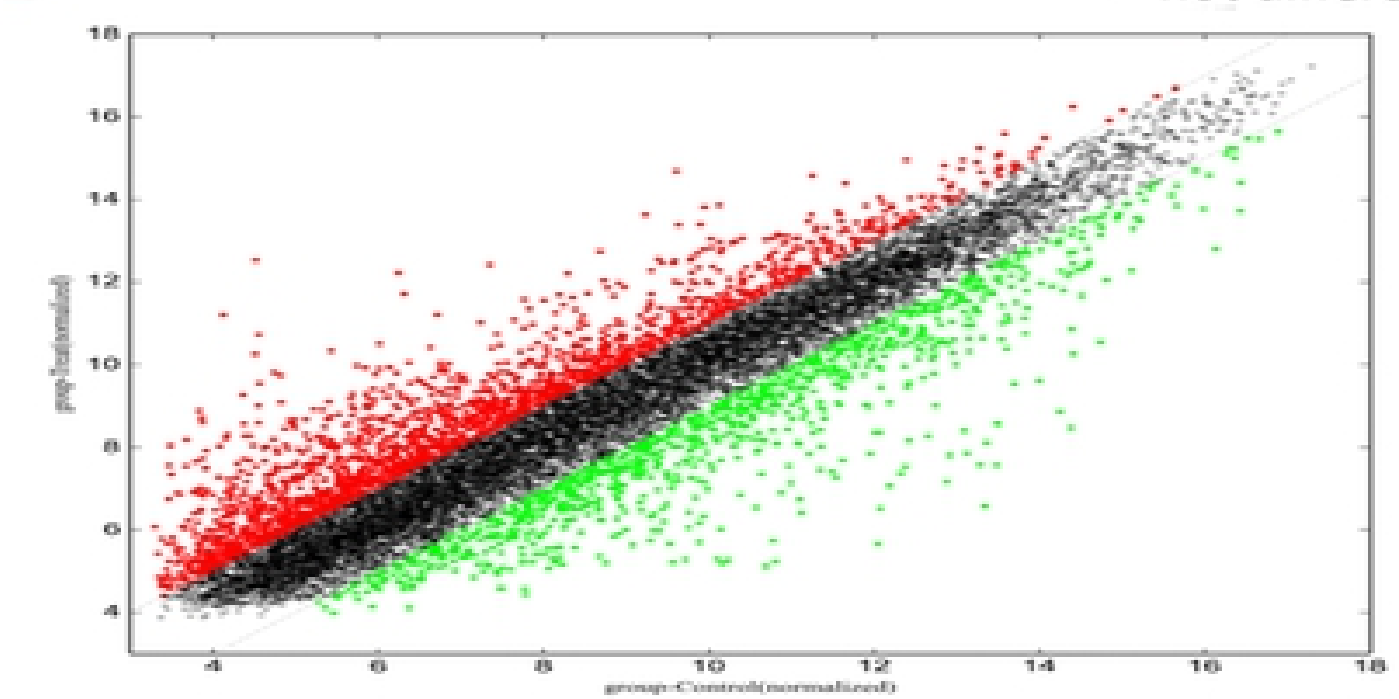

- down-regulated

Color Key

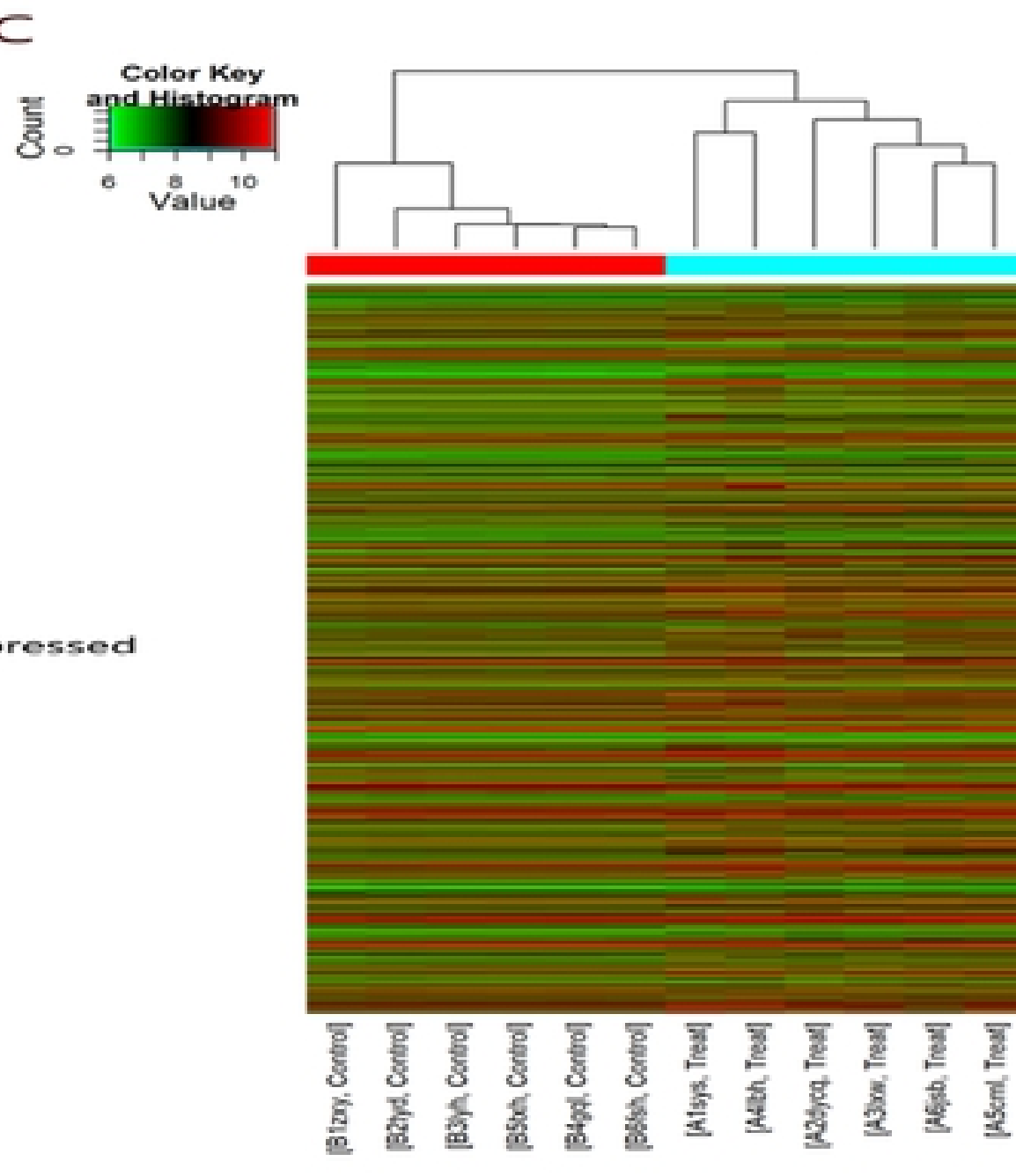

- value ${ }^{10}$
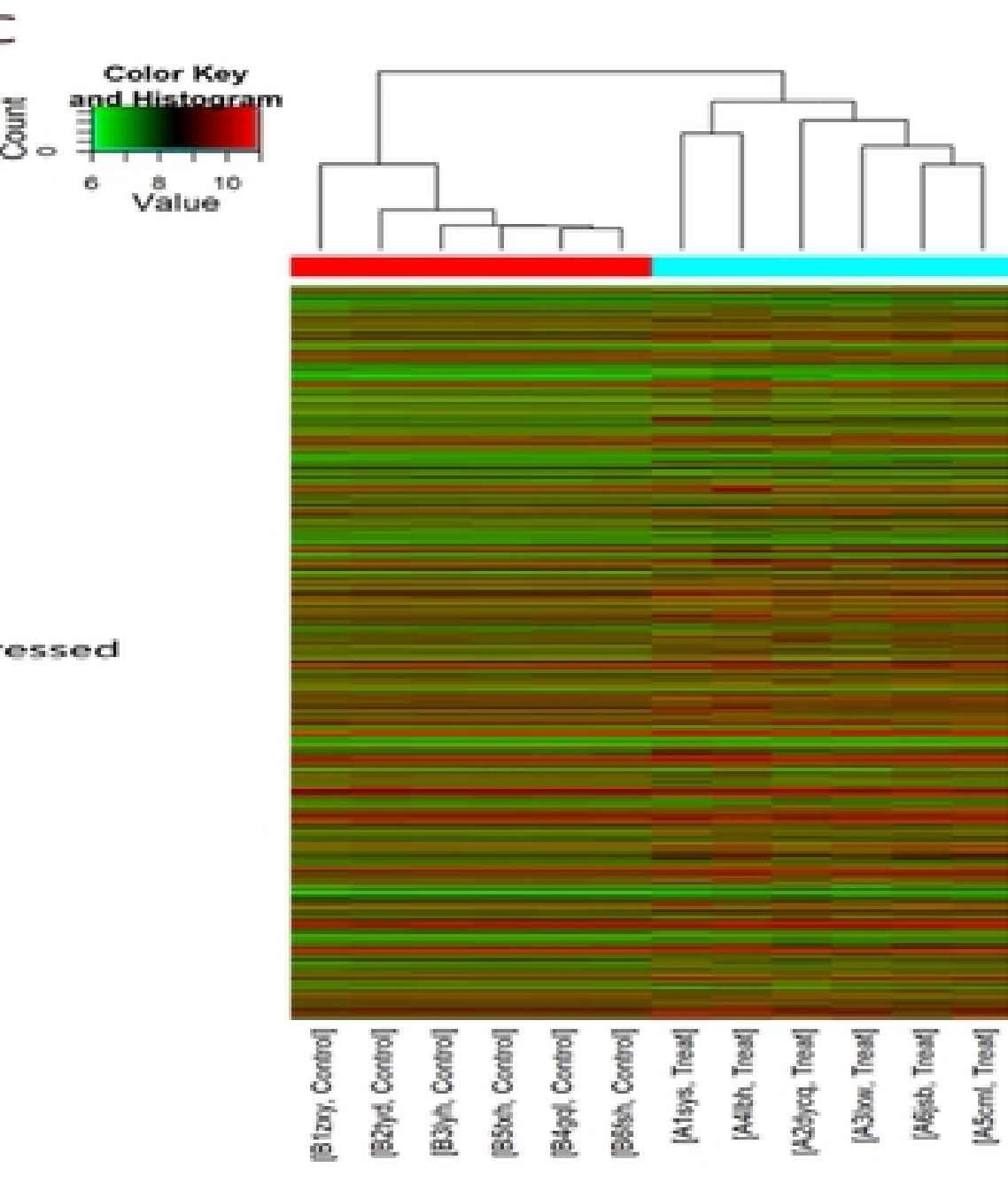

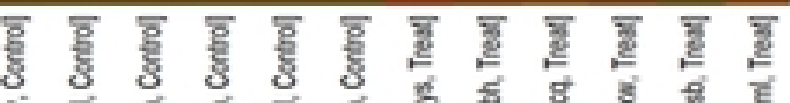

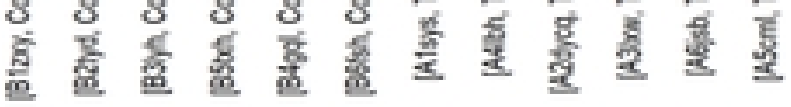

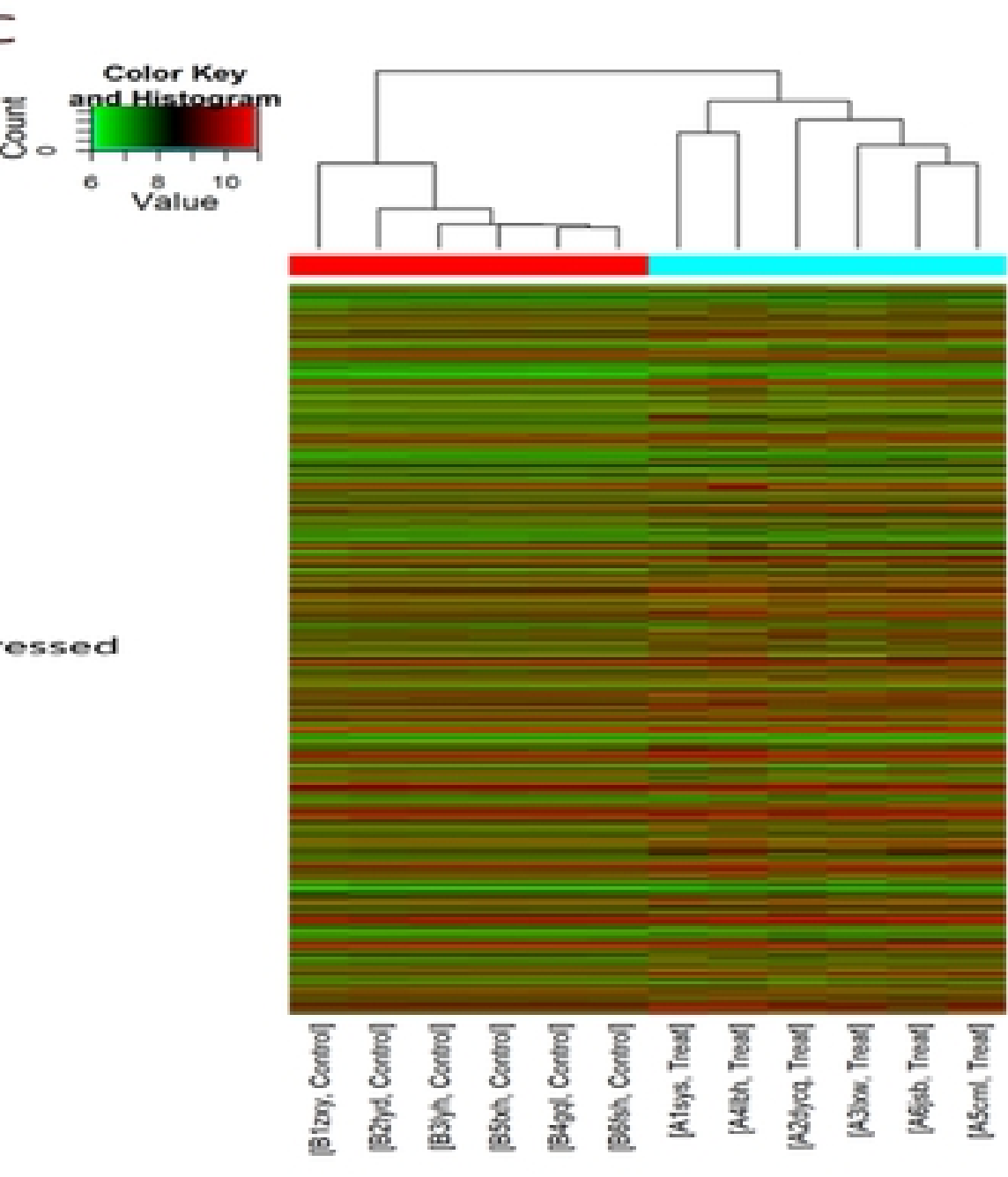

Figure1 
Sig pathway of DE gene

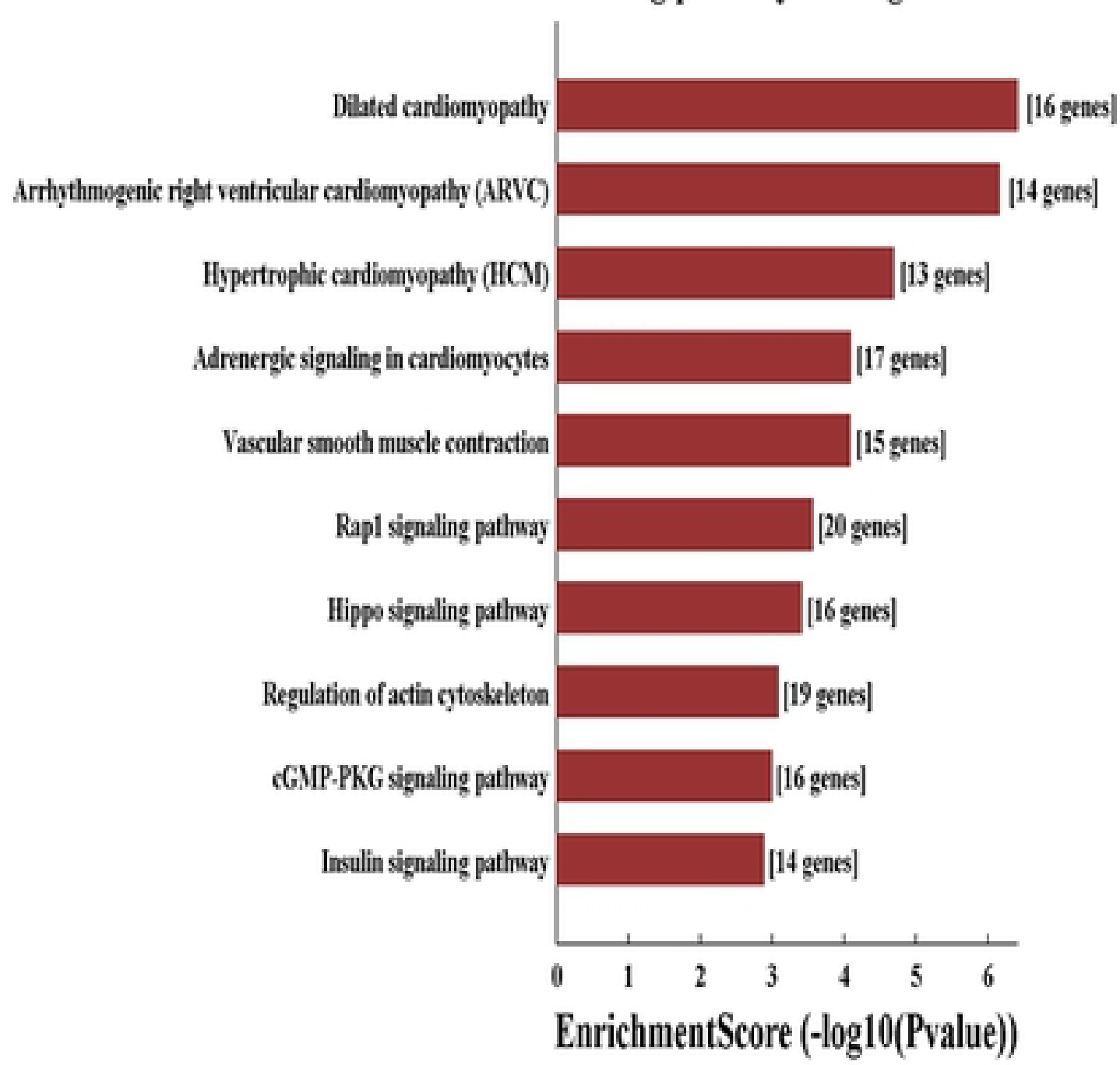

Sig pathway of DE gene

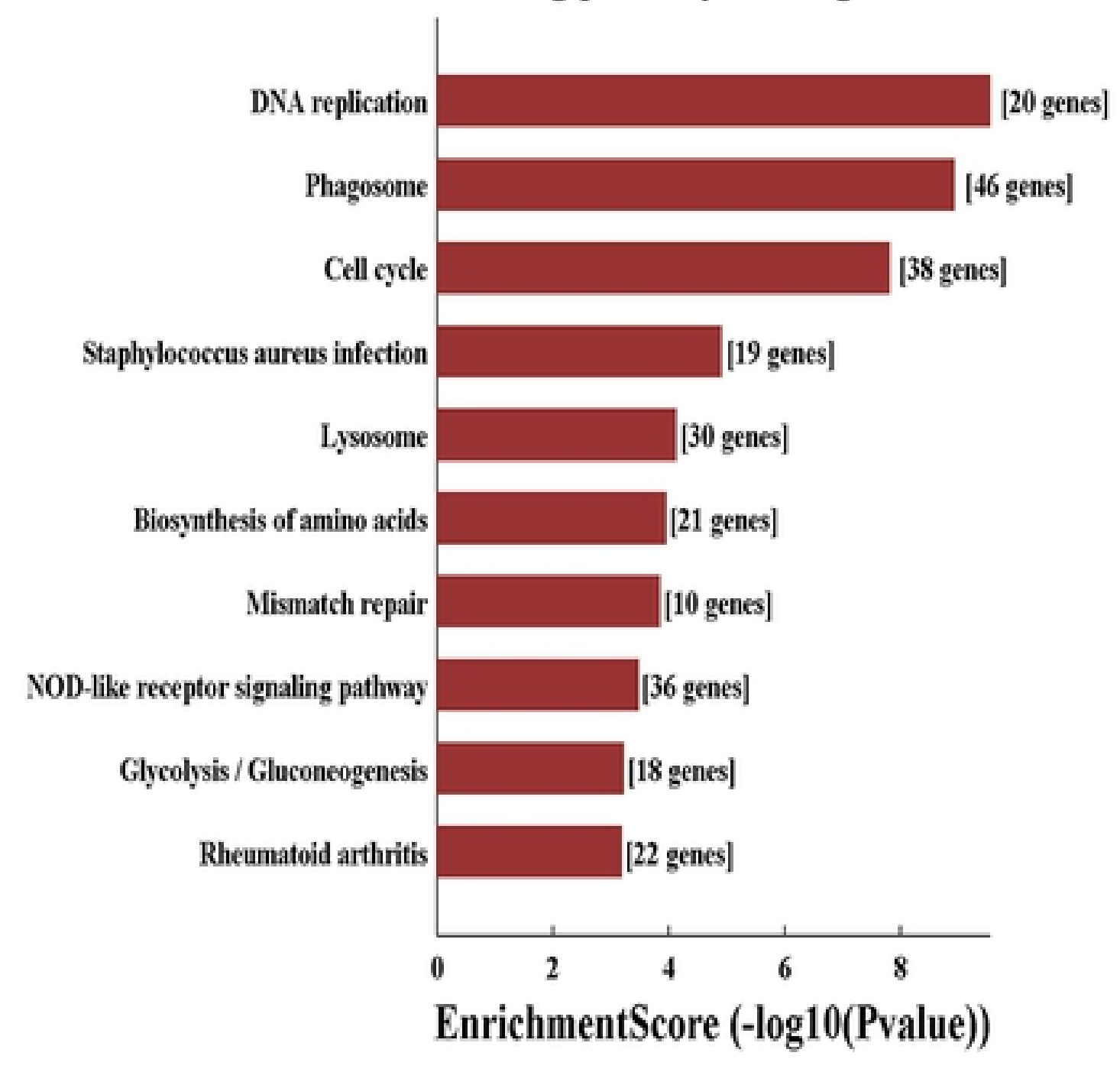

Figure4 
snt

NFI4 NUSAP1

HLA.DMA KIF 15 -

ARHGAS11A X 0 CA Trk 9 ....... Can

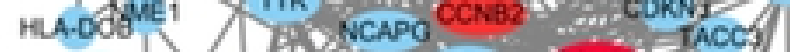

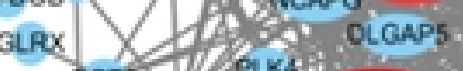

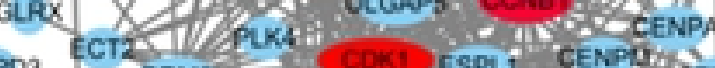
TESPLi " CENPBH CENP ITA TRMME RNF123 Coc4s RNF123 COC44 CDC6

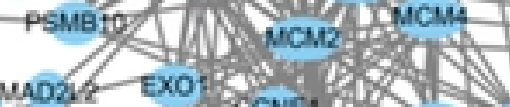
HeLIS: TENO STMES

GINSA os

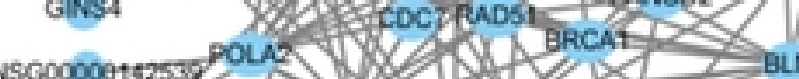

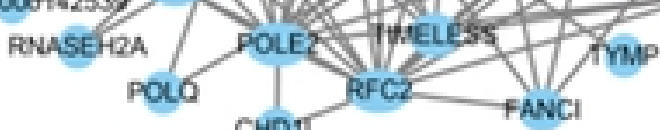
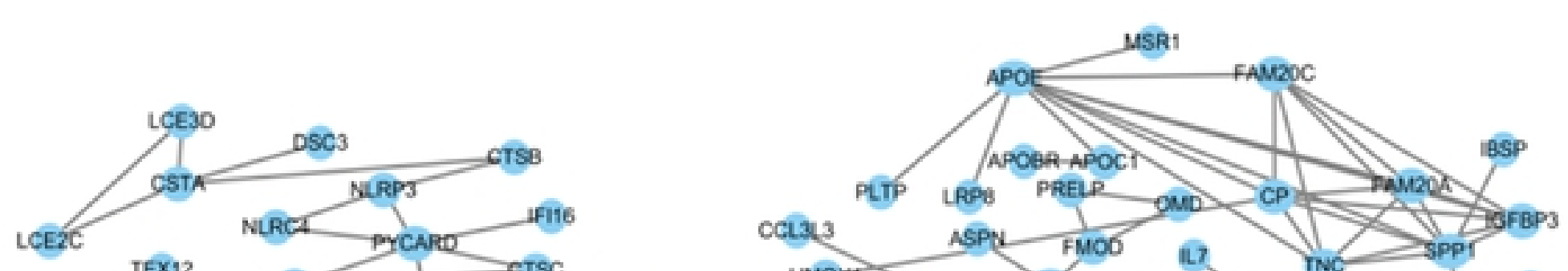

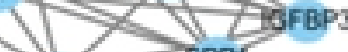

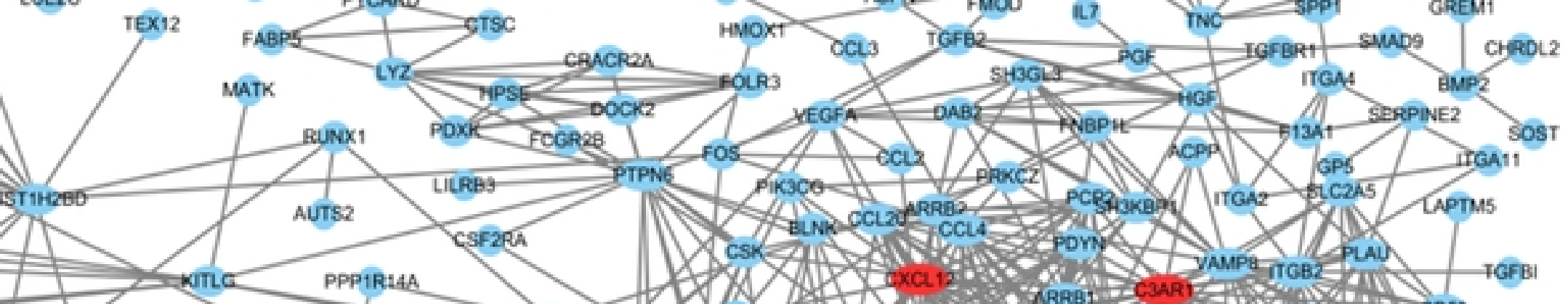

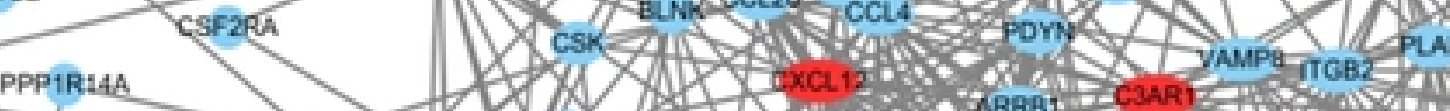

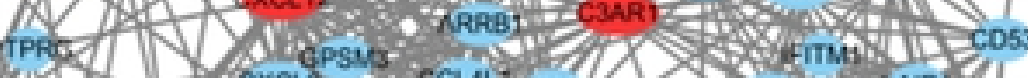
ZFPM1
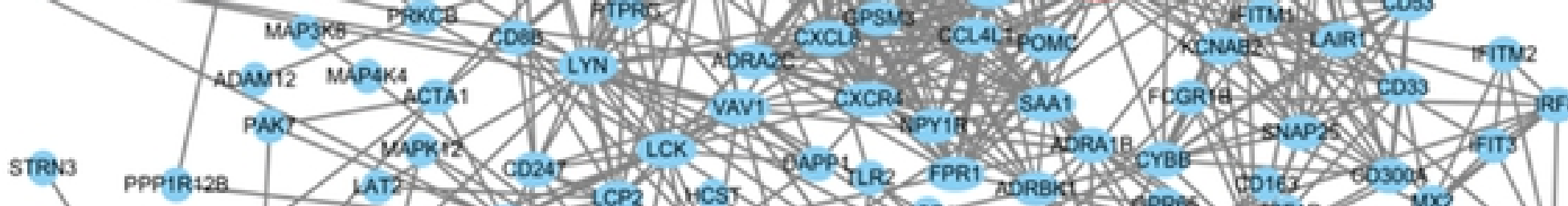
MTSS1 HAT27

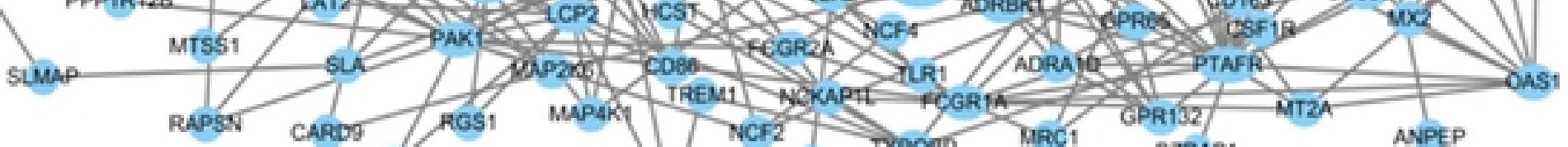
eYs SKAP2 CLECZA DYROEP MAC1 GZRAP

\section{Figure5}


Module 1

Module 2

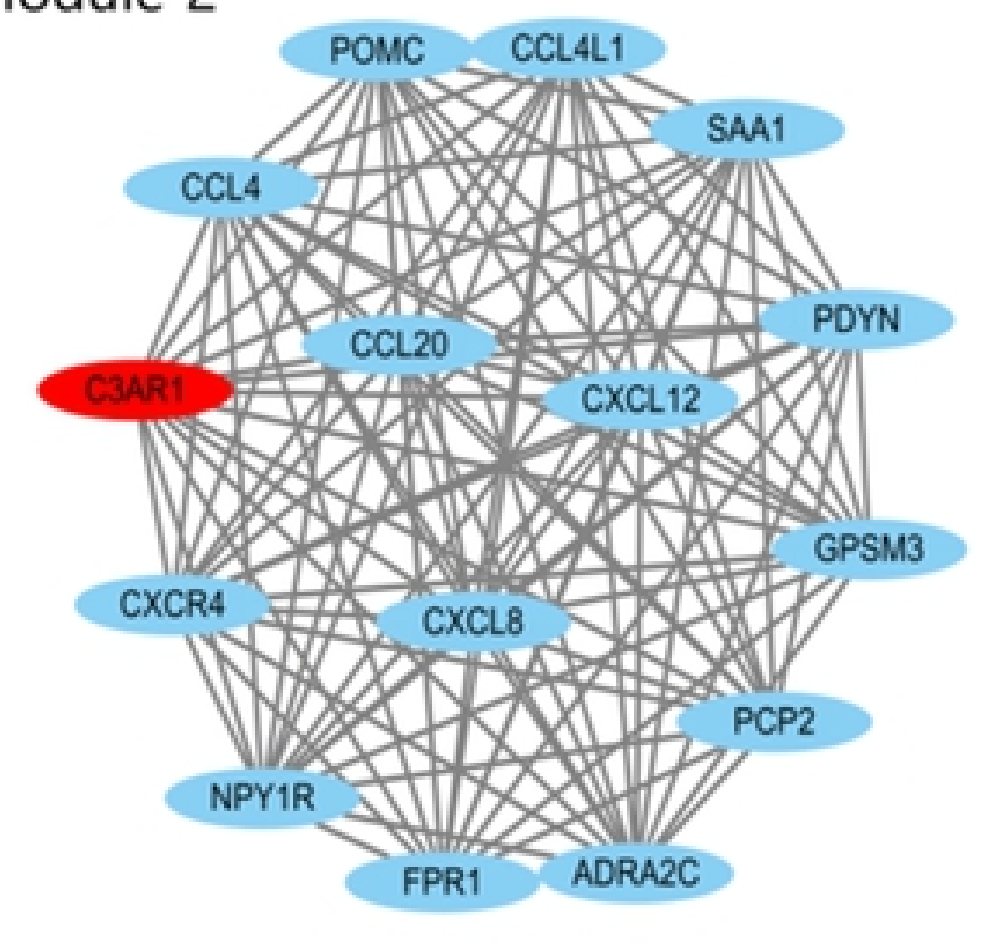

Module 3 KCNAB2

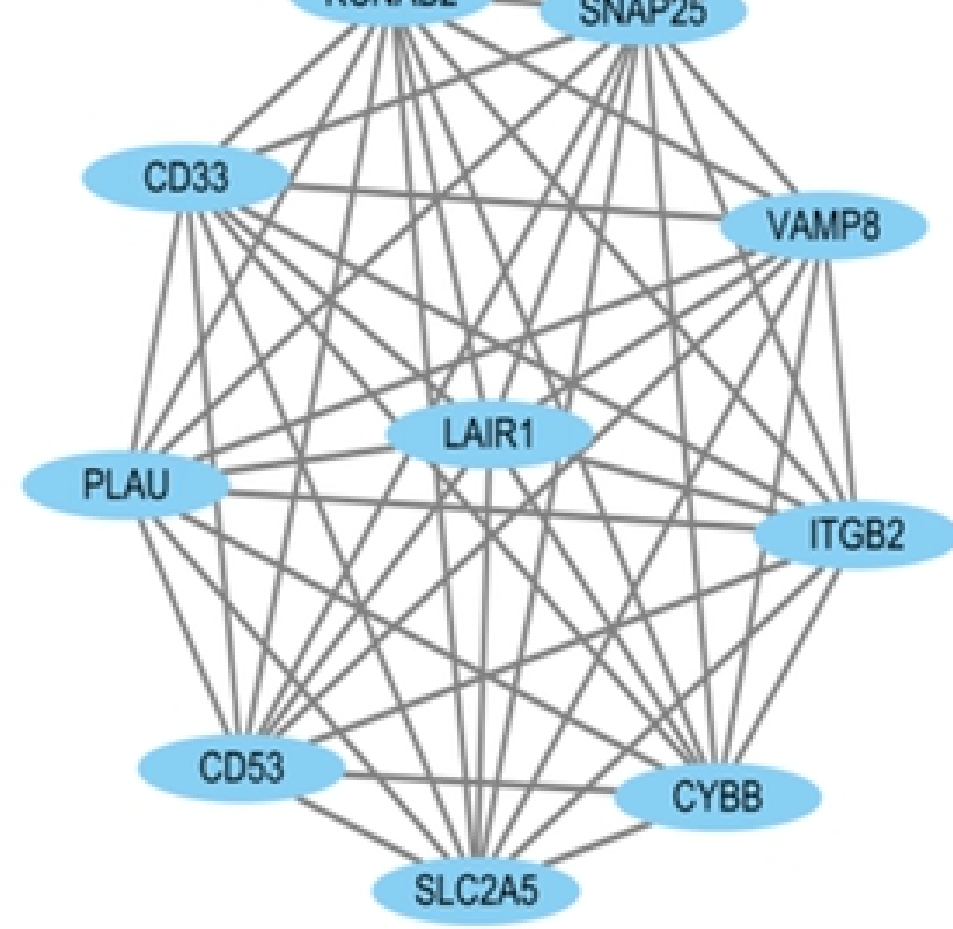

Figure6 
Validation of mRNA microarray results by real-time qPCR

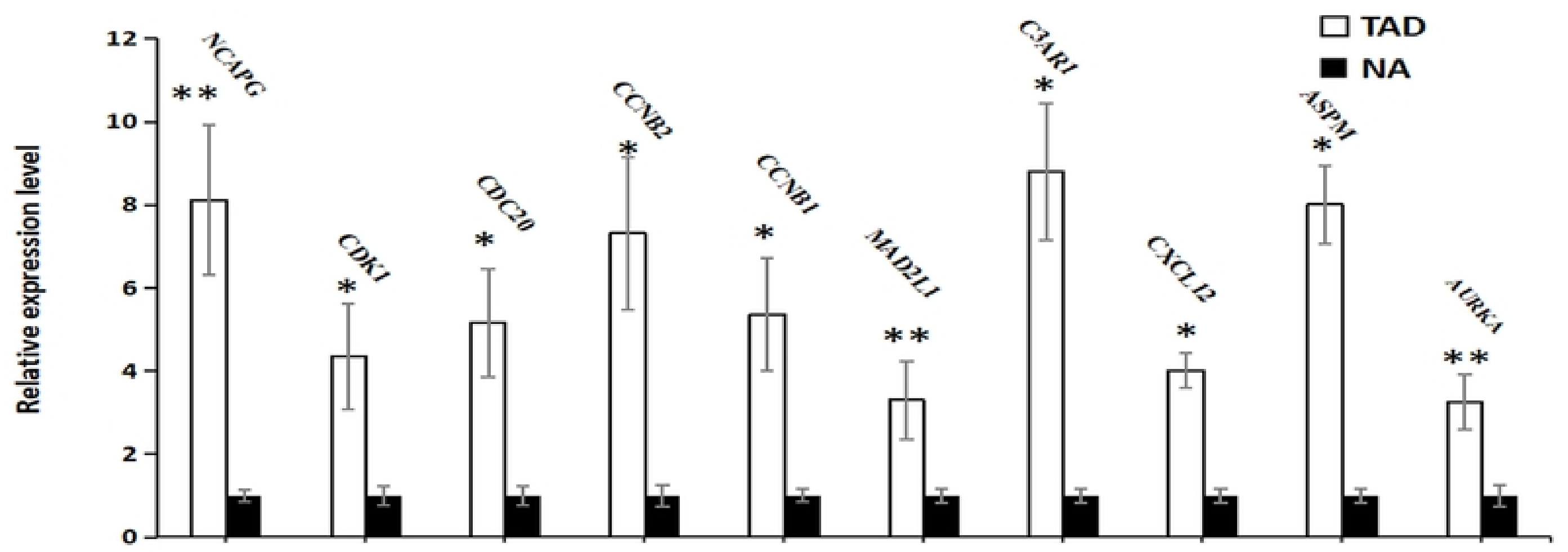

mRNAs, $2-\triangle \triangle C T$

( 10 thoracic aortic dissection patients and 10 none thoracic aortic dissection patients )

Figure7 
- Biological Processe

- Cellular Component

- Molecular Function

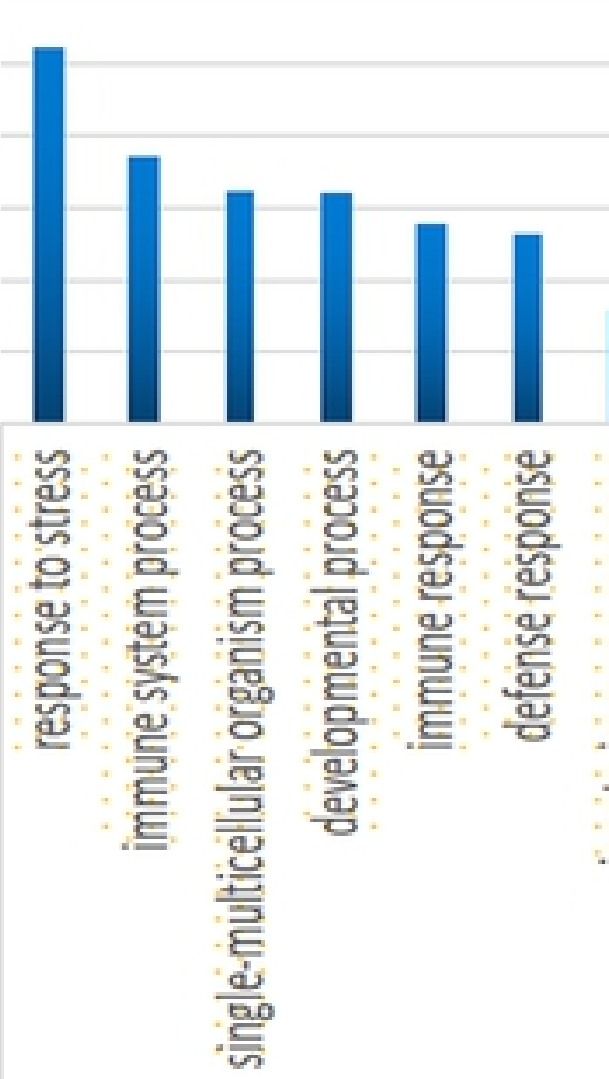

BP
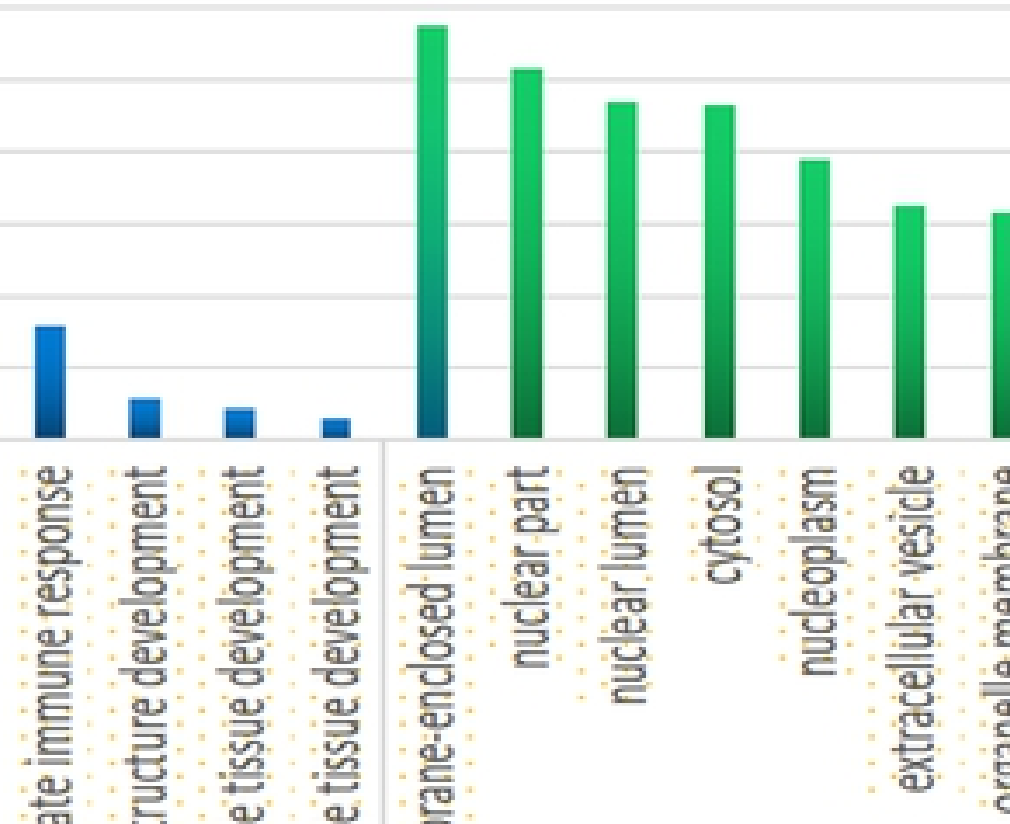

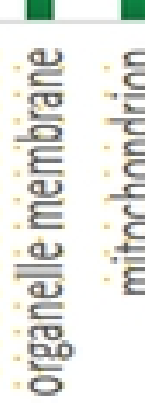

$\mathrm{CC}$

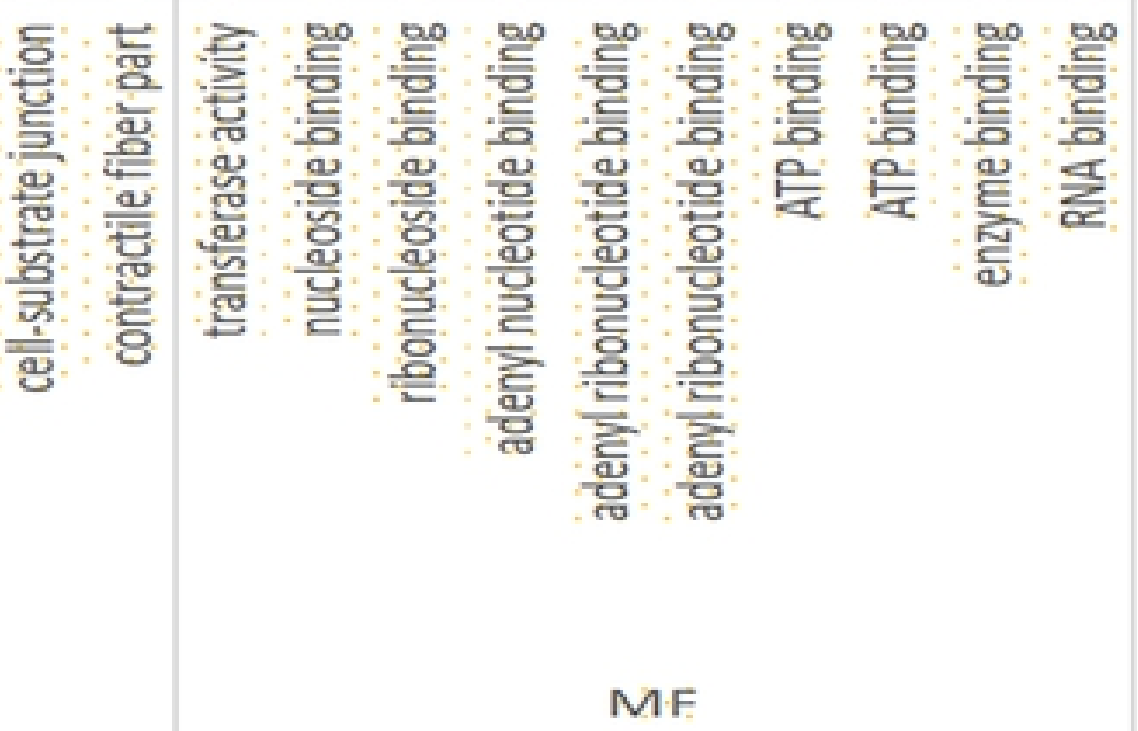

Figure2 
Vascular smooth muscle contraction

staphylococcus aureus infection -

Rheumatoid arthritis

Regulation of actin cytosketeton

Rap 1 signaling pathway

Phagosome

NOD-like receptor signaling pathway

Mismatch repair

Insulin signaling pathway

Hypertrophic cardiomyopathy (HCM) -

Hippo signaling pathway

Glycolysis / Gluconeogenesis DNA replication - Homo sapiens Dilated cardiomyopathy CGMP-PKG signaling pathway

Cell cycre Adrenergic signaling in cardiomyocytes

$$
0 e+00
$$

$-\log _{10}(P V a l u e)$

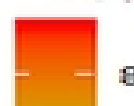

8

6

4

Gene number

- 10

- 20

30

\section{Figure3}

